Research Paper

\title{
A 10-year Population-based Study of the Differences between NECs and Carcinomas of the Esophagus in Terms of Clinicopathology and Survival
}

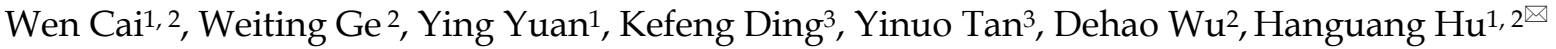 \\ 1. Department of Medical Oncology, the Second Affiliated Hospital, School of Medicine, Zhejiang University, Hangzhou, Zhejiang, China \\ 2. Cancer Institute (Key Laboratory of Cancer Prevention and Intervention, China National Ministry of Education), the Second Affiliated Hospital, School of \\ Medicine, Zhejiang University, Hangzhou, Zhejiang, China \\ 3. Department of Surgical Oncology, the Second Affiliated Hospital, School of Medicine, Zhejiang University, Hangzhou, Zhejiang, China
}

$\square$ Corresponding author: Department of Medical Oncology, the Second Affiliated Hospital, School of Medicine, Zhejiang University, Hangzhou, Zhejiang, China. Tel: +86 13858110651; Fax: +86 571 87767088; Email: huhanguang@zju.edu.cn

(C) Ivyspring International Publisher. This is an open access article distributed under the terms of the Creative Commons Attribution (CC BY-NC) license (https://creativecommons.org/licenses/by-nc/4.0/). See http://ivyspring.com/terms for full terms and conditions.

Received: 2018.08.26; Accepted: 2019.01.14; Published: 2019.02.26

\begin{abstract}
Purpose: The prevalence of esophageal NECs is rising, but to date, no studies have compared its clinicopathological characteristics to those of esophageal ACs and SCCs from the same period.

Patients and methods: A 10-year population-based retrospective cohort study was conducted with the Surveillance, Epidemiology, and End Results Program database. Statistical analyses were performed using Intercooled Stata 12.0 software.

Results: A total of 17,196 eligible patients with esophageal tumors, including 246 NECs, 6,102 SCCs and 10,848 ACs, were analyzed. ACs showed an obviously higher prevalence than the other two tumor types, and the prevalence of NECs was increasing. NECs were associated with an obviously worse survival than ACs (log-rank test, $P<0.01$ ). Most NECs were poorly differentiated and had an obviously higher percentage of metastasis. NECs and ACs often metastasized to the liver (29.41\% and $23.11 \%$, respectively), while SCCs typically metastasized to the lung $(15.84 \%)$ and distant lymph nodes (15.37\%). We divided the patients into two groups for further analysis according to the metastasis status. For NECs, no benefit was obtained by surgery in metastatic disease. For SCCs and ACs, surgery of the primary sites produced survival benefits in both groups, but the benefits of lymphadenectomy and metastasis dissection need further study.

Conclusion: NECs of the esophagus have the worst prognosis compared to SCCs and ACs from the same period. Radical surgery provides limited benefits to patients diagnosed with NECs, so systemic treatments should be considered instead of surgical procedures. A unique guideline with a new staging and grading system for esophageal NECs is urgently needed.
\end{abstract}

Key words: esophageal cancer, neuroendocrine carcinoma, clinicopathological differences, adenocarcinoma, squamous cell carcinoma

\section{Introduction}

Esophageal cancer is the $7^{\text {th }}$ most common cause of cancer death worldwide and is more common in men ${ }^{1}$. Esophageal cancer is endemic in many parts of the world, particularly in developing nations, where the disease is among the top five most common causes of cancer death ${ }^{2,3}$. In 2015, an estimated 16,980 people will be diagnosed with esophageal cancer, and 15,590 people will eventually die of the disease in the United States ${ }^{4,5}$.

Squamous cell carcinoma (SCC) and adenocarcinoma (AC) are two most common pathological types of esophageal cancer. In 2012, Arnold M and colleagues reported the global incidence of esophageal cancer, indicating that approximately $87 \%$ of all 
esophageal cancers worldwide were SCCs, with the highest incidence rates in populations within SouthEastern and Central Asia; only $11 \%$ of all esophageal cancers are ACs, with an elevated burden seen in Northern and Western Europe, North America, and Oceania $^{6}$.

Although SCC and AC combine to represent the vast majority of esophageal cancers, esophageal neuroendocrine carcinoma (NEC), which was reported in 1952 by a British pathologist named Dr. McKeon $^{7}$ and classified into small and large cell types by the WHO, has been gradually recognized by scientists and clinicians. NECs exhibit biological behaviors that are different from those of other esophageal cancers, but clinicians lack NEC treatment experience and suitable guidelines. Additionally, data describing NECs not only in terms of epidemiology but also regarding clinicopathologic features are lacking due to the low incidence of the disease. Some case reports, small series, and reviews have speculated on the incidence of NECs, which vary geographically to some extent, accounting for $0.5 \%$ to $5.9 \%$ of all esophageal cancers in Chinese patients ${ }^{8-11}$, $0.8 \%$ to $2.8 \%$ in Japanese patients ${ }^{12,13}$ and $1 \%$ to $2.8 \%$ in western patients ${ }^{14}, 15$. However, few investigations of esophageal NECs have been conducted.

To the best of our knowledge, no well-known study has compared the differences between NECs, SCCs and ACs. Therefore, we retrieved data on these esophageal cancer types from the Surveillance, Epidemiology, and End Results (SEER) database and elucidated the distinctions in terms of the epidemiology, clinicopathologic features and risk factors that contributed to cancer mortality.

\section{Material and Methods}

\section{Data source}

Data were retrieved from the SEER database based on the November 2016 submission for cases with tumors of malignant behavior and based on known patient age for cases diagnosed between the inception of the SEER program in 1973 until 2014. All cases of esophageal carcinomas had tumor site codes (C15.0-C15.9) and ICD-9 codes and were diagnosed between 2004 and 2014. Poorly differentiated esophageal carcinomas were identified using the International Classification of Diseases for Oncology, 3rd Edition (ICD-O-3) morphology codes. These codes corresponded to the following clinical/histological diagnoses: NEC (8013, 8041 and 8246); SCC (8051, 8070, 8074, 8077, 8083, and 8560); and AC (8140, 8148, 8200,8244 , and 8430).

The data we used to analyze the metastasis patterns of the different pathological types were only from 2010 to 2014 because the specific records for the metastatic sites in the SEER database started from 2010.

We excluded cases if 1) multiple primary tumors were evident; 2) the survival data description was incomplete; 3) the surgical data description was incomplete; or 4) the pathological type was unspecific.

\section{Statistical analyses}

We obtained SEER frequency and survival data using SEER ${ }^{*}$ Stat software, version 8.3.4. The study population was divided according to the year of diagnosis and pathological type. Mean and median values were used to describe continuous data, with discrete variables displayed as totals and frequencies. Overall median survival and survival rates were calculated. We analyzed the metastasis patterns by counting the patients' metastasis site records at the time of diagnosis regardless of whether multiple metastases were present. The patients' demographic and tumor characteristics were summarized with descriptive statistics. Comparisons of categorical variables among the different groups of patients were performed using the Chi square test. Deaths attributed to esophageal carcinomas were treated as events. Survival function estimations and comparisons among different variables were performed using the Kaplan-Meier method and compared using the logrank test. The Cox proportional hazards model was used to compare the effects of prognostic variables on survival. All statistical analyses were performed using Intercooled Stata 12.0 (Stata Corporation, College Station, TX, USA). Statistical significance was considered when the two-sided $\mathrm{P}$ value $<0.05$.

\section{Results}

\section{Patient characteristics and the 10-year prevalence}

Using the SEER database, a total of 17,196 patients diagnosed with esophageal carcinomas between 2004-2014, including 246 NEC patients, 6102 SCC patients and 10848 AC patients, met our inclusion criteria. We have summarized the selection process in Fig. S1. The details of the patients' basic characteristics based on the pathological type of the tumor are shown in Table S1. The mean age at diagnosis seemed similar (NECs: 66.65-year-old, SCCs: 67.19-year-old, ACs: 66.10-year-old). The included clinicopathologic features of the carcinoma types were so different that the results of the Chi-square test were all significant $(P<0.01)$. NECs often originated from lower third of the esophagus $(53.85 \%)$, as did the ACs $(77.76 \%)$, while the middle third of the esophagus was the common primary site 
of the SCCs (38.76\%). The NEC sizes at diagnosis were larger than the sizes of the other two types. The metastasis statuses and common metastasis sites of the three kinds of carcinomas were completely different. The NECs showed obviously higher rates of metastasis disease $(62.20 \%)$, whereas $58.72 \%$ of SCCs and $46.66 \%$ of ACs were not metastatic. Esophageal ACs, SCCs and NECs combined to represent almost all esophageal cancers in the study. We calculated the 10-year duration prevalence of these three main kinds of pathological types, whereby the populations were estimated by averaging the 2013 and 2014 populations. The prevalence of the ACs was obviously higher prevalence than those of the other two kinds, and the prevalence of the NECs were increasing (Fig. S2).
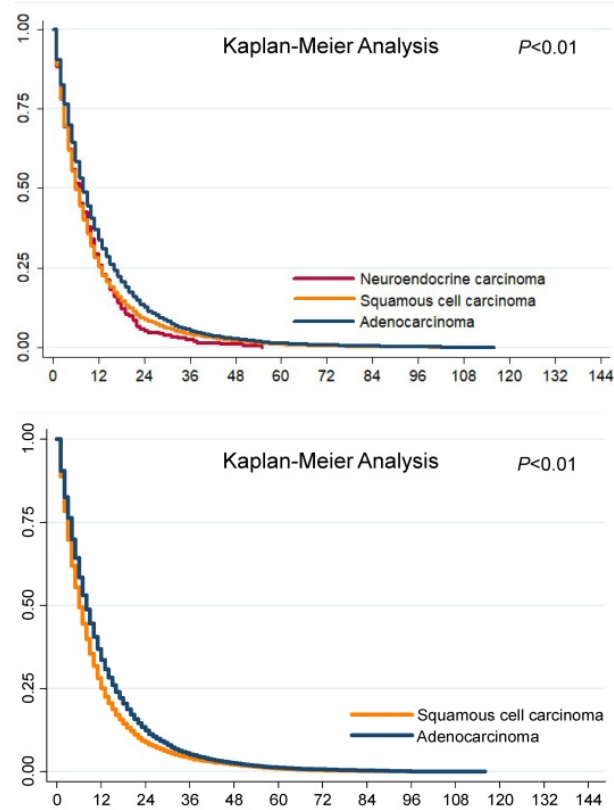

Figure 1. Survival analysis of esophageal cancer in different pathological types.
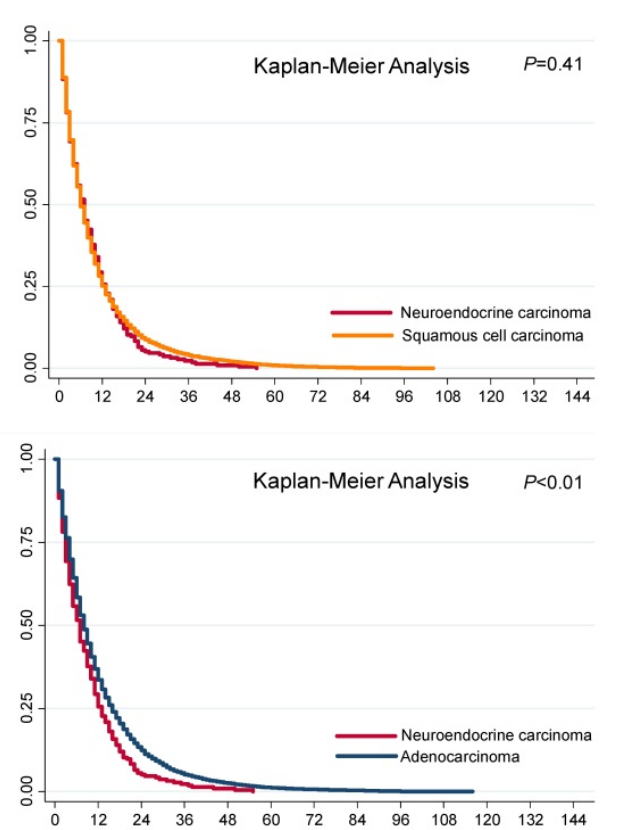
survival. using Kaplan-Meier estimates. We compared the groups in pairs using the log-rank test. The results showed that no significant difference was evident between the SCCs $(P=0.41)$ and NECs, but both the survival rates of the SCCs and NECs were worse than that of the ACs $(P<0.01)$. We further divided the patients into metastasis and non-metastasis groups and estimated the overall survival rates for analysis in the current study. The 1-year, 3-year and 5-year overall survival rates are presented in Fig. 2. Metastatic disease showed an obviously worse

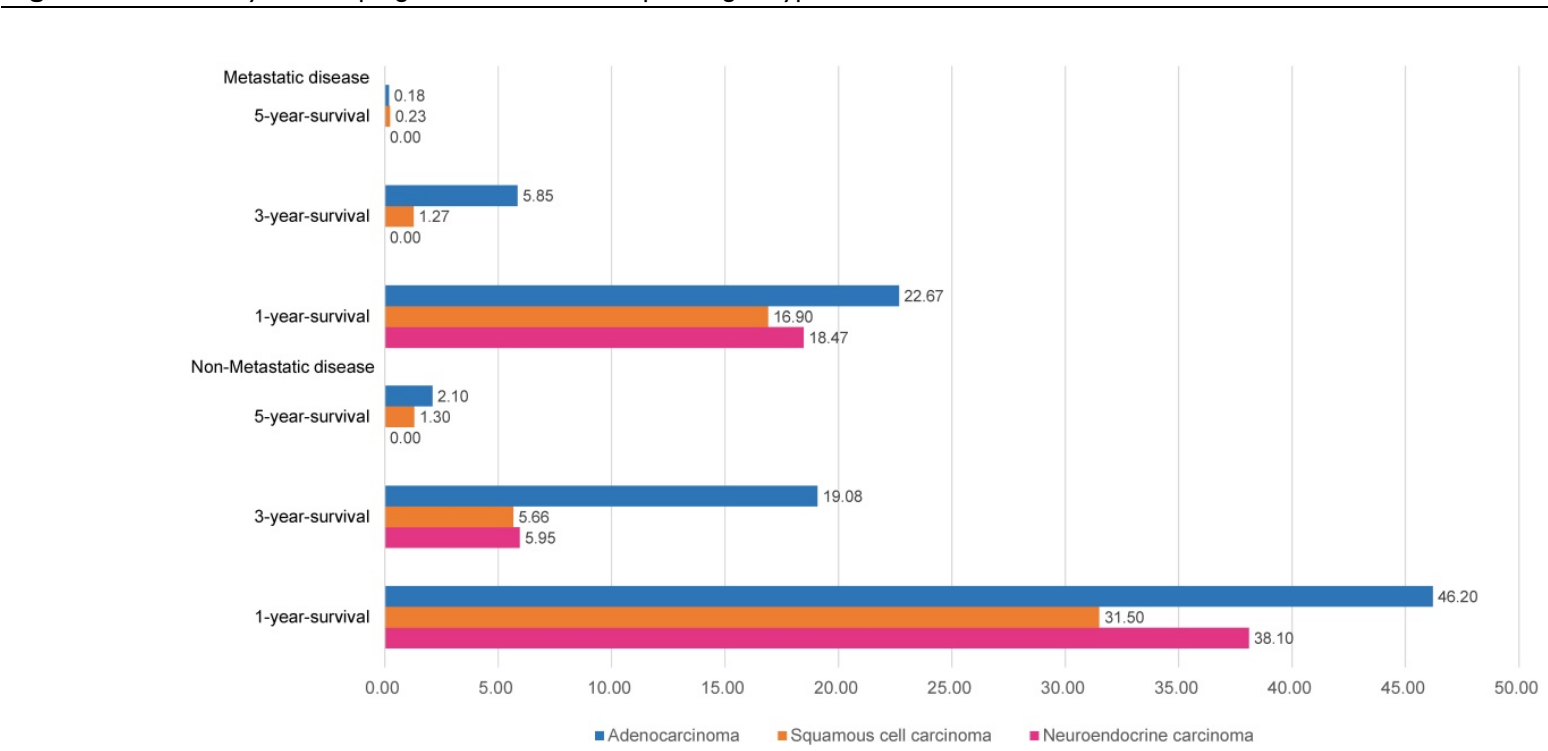

Figure 2. Year-specific overall survival rate in different pathological types. 
Table 1. Risk factors of non-metastasis disease

\begin{tabular}{|c|c|c|c|c|c|c|c|c|c|c|c|c|}
\hline & \multicolumn{4}{|c|}{ Neuroendocrine carcinoma } & \multicolumn{4}{|c|}{ Squamous cell carcinoma } & \multicolumn{4}{|c|}{ Adenocarcinoma } \\
\hline & \multicolumn{2}{|c|}{ Univariate analyses } & \multicolumn{2}{|c|}{ Multivariate analyses } & \multicolumn{2}{|c|}{ Univariate analyses } & \multicolumn{2}{|c|}{ Multivariate analyses } & \multicolumn{2}{|c|}{ Univariate analyses } & \multicolumn{2}{|c|}{ Multivariate analyses } \\
\hline & $\begin{array}{l}\text { HR } \\
(95 \% \mathrm{CI})\end{array}$ & $\begin{array}{l}P \text { - } \\
\text { value }\end{array}$ & $\begin{array}{l}\text { HR } \\
(95 \% \mathrm{CI})\end{array}$ & $\begin{array}{l}\mathrm{P}- \\
\text { value }\end{array}$ & $\begin{array}{l}\text { HR } \\
(95 \% \mathrm{CI})\end{array}$ & $\begin{array}{l}P \text { - } \\
\text { value }\end{array}$ & $\begin{array}{l}\text { HR } \\
(95 \% \mathrm{CI})\end{array}$ & $\begin{array}{l}\text { P- } \\
\text { value }\end{array}$ & $\begin{array}{l}\text { HR } \\
(95 \% \mathrm{CI})\end{array}$ & $\begin{array}{l}\mathrm{P}- \\
\text { value }\end{array}$ & $\begin{array}{l}\text { HR } \\
(95 \% C I)\end{array}$ & $\begin{array}{l}\text { P- } \\
\text { value }\end{array}$ \\
\hline \multicolumn{13}{|l|}{ Gender } \\
\hline Female & reference & & reference & & reference & & reference & & reference & & reference & \\
\hline Male & $1.07(0.65-1.75)$ & 0.79 & $1.16(0.50-2.70)$ & 0.73 & $1.01(0.94-1.09)$ & 0.69 & $1.01(0.94-1.09)$ & 0.70 & $0.82(0.75-0.89)$ & $<0.01$ & $0.92(0.84-1.00)$ & 0.04 \\
\hline \multicolumn{13}{|l|}{ Age } \\
\hline$<66$ year-old & reference & & reference & & reference & & reference & & reference & & reference & \\
\hline$>=66$ year-old & $1.31(0.80-2.14)$ & 0.28 & $1.38(0.78-2.44)$ & 0.27 & $1.17(1.09-1.25)$ & $<0.01$ & $1.13(1.05-1.22)$ & $<0.01$ & $1.30(1.23-1.37)$ & $<0.01$ & $1.13(1.06-1.20)$ & $<0.01$ \\
\hline \multicolumn{13}{|l|}{ Location } \\
\hline $\begin{array}{l}\text { Upper third of } \\
\text { esophagus }\end{array}$ & reference & & reference & & reference & & reference & & reference & & reference & \\
\hline $\begin{array}{l}\text { Middle third of } \\
\text { esophagus }\end{array}$ & $0.70(0.28-1.75)$ & 0.44 & $0.10(0.02-0.41)$ & $<0.01$ & $0.95(0.87-1.05)$ & 0.35 & $1.04(0.95-1.15)$ & 0.39 & $1.33(1.03-1.73)$ & 0.03 & $1.59(1.22-2.06)$ & $<0.01$ \\
\hline $\begin{array}{l}\text { Lower third of } \\
\text { esophagus }\end{array}$ & $0.90(0.37-2.15)$ & 0.81 & $0.20(0.05-0.85)$ & 0.03 & $1.00(0.90-1.11)$ & 0.98 & $1.15(1.03-1.28)$ & 0.01 & $1.15(0.90-1.46)$ & 0.26 & $1.50(1.18-1.92)$ & $<0.01$ \\
\hline Unspecific & $2.19(0.77-6.26)$ & 0.14 & $0.30(0.06-1.41)$ & 0.13 & $1.15(1.01-1.30)$ & 0.03 & $1.21(1.07-1.37)$ & $<0.01$ & $1.40(1.08-1.80)$ & 0.01 & $1.58(1.22-2.05)$ & $<0.01$ \\
\hline \multicolumn{13}{|l|}{ Grade } \\
\hline I & reference & & reference & & reference & & reference & & reference & & reference & \\
\hline II & NA & & NA & & $1.03(0.86-1.23)$ & 0.75 & $0.96(0.80-1.15)$ & 0.68 & $1.08(0.93-1.25)$ & 0.32 & $1.07(0.92-1.25)$ & 0.35 \\
\hline III & NA & & NA & & $1.02(0.85-1.22)$ & 0.81 & $0.98(0.82-1.17)$ & 0.80 & $1.29(1.11-1.49)$ & $<0.01$ & $1.29(1.11-1.49)$ & $<0.01$ \\
\hline IV & $1.40(0.76-2.58)$ & 0.28 & $3.05(1.32-7.07)$ & 0.01 & $0.89(0.59-1.34)$ & 0.57 & $0.77(0.51-1.17)$ & 0.22 & $1.10(0.81-1.50)$ & 0.53 & $1.21(0.89-1.64)$ & 0.23 \\
\hline Unspecific & $0.61(0.32-1.19)$ & 0.15 & $0.66(0.25-1.73)$ & 0.40 & $1.00(0.83-1.21)$ & 0.98 & $0.90(0.74-1.08)$ & 0.26 & $1.22(1.04-1.43)$ & 0.02 & $1.08(0.92-1.27)$ & 0.34 \\
\hline \multicolumn{13}{|l|}{ Tumorsize } \\
\hline$<2 \mathrm{~cm}$ & reference & & reference & & reference & & reference & & reference & & reference & \\
\hline $2-3 \mathrm{~cm}$ & $1.83(0.55-6.12)$ & 0.33 & $1.09(0.26-4.48)$ & 0.91 & $1.15(0.96-1.37)$ & 0.14 & $1.11(0.92-1.33)$ & 0.27 & $1.18(1.03-1.35)$ & 0.02 & $1.22(1.06-1.39)$ & 0.01 \\
\hline $3-4 \mathrm{~cm}$ & $2.04(0.59-7.14)$ & 0.26 & $1.97(0.42-9.13)$ & 0.39 & $1.23(1.04-1.46)$ & 0.02 & $1.16(0.98-1.39)$ & 0.09 & $1.18(1.04-1.35)$ & 0.01 & $1.15(1.00-1.31)$ & 0.05 \\
\hline $4-5 \mathrm{~cm}$ & $2.56(0.60-10.95)$ & 0.21 & $1.93(0.35-10.68)$ & 0.45 & $1.32(1.12-1.56)$ & $<0.01$ & $1.18(0.99-1.40)$ & 0.06 & $1.20(1.05-1.37)$ & 0.01 & $1.08(0.95-1.24)$ & 0.24 \\
\hline $5-6 \mathrm{~cm}$ & $3.75(0.97-14.50)$ & 0.06 & $3.64(0.76-17.38)$ & 0.11 & $1.34(1.12-1.60)$ & $<0.01$ & $1.16(0.96-1.39)$ & 0.12 & $1.29(1.12-1.49)$ & $<0.01$ & $1.18(1.02-1.37)$ & 0.03 \\
\hline $6-7 \mathrm{~cm}$ & $3.00(0.84-10.68)$ & 0.09 & $2.05(0.40-10.44)$ & 0.39 & $1.34(1.10-1.64)$ & $<0.01$ & $1.19(0.97-1.45)$ & 0.10 & $1.41(1.20-1.67)$ & $<0.01$ & $1.26(1.06-1.49)$ & 0.01 \\
\hline $7-8 \mathrm{~cm}$ & $4.00(0.92-17.35)$ & 0.06 & $1.86(0.31-11.25)$ & 0.50 & $1.45(1.17-1.79)$ & $<0.01$ & $1.26(1.02-1.57)$ & 0.04 & $1.39(1.16-1.66)$ & $<0.01$ & $1.23(1.03-1.48)$ & 0.02 \\
\hline$>8 \mathrm{~cm}$ & $4.64(1.53-14.09)$ & 0.01 & $3.77(1.04-13.68)$ & 0.04 & $1.65(1.38-1.98)$ & $<0.01$ & $1.43(1.19-1.73)$ & 0.00 & $1.56(1.34-1.82)$ & $<0.01$ & $1.41(1.21-1.65)$ & $<0.01$ \\
\hline Unspecific & $3.09(1.17-8.11)$ & 0.02 & $2.73(0.85-8.75)$ & 0.09 & $1.32(1.15-1.52)$ & $<0.01$ & $1.09(0.94-1.26)$ & 0.23 & $1.44(1.30-1.61)$ & $<0.01$ & $1.14(1.02-1.27)$ & 0.02 \\
\hline \multicolumn{13}{|l|}{ Tstage } \\
\hline T0 & reference & & reference & & reference & & reference & & reference & & reference & \\
\hline $\mathrm{T} 1$ & NA & & NA & & $3.20(0.80-12.82)$ & 0.10 & $2.63(0.65-10.68)$ & 0.18 & NA & & NA & \\
\hline $\mathrm{T} 2$ & $2.23(0.87-5.71)$ & 0.10 & $4.39(1.16-16.54)$ & 0.03 & $2.62(0.65-10.53)$ & 0.18 & $2.31(0.57-9.38)$ & 0.24 & $0.82(0.74-0.90)$ & $<0.01$ & $0.84(0.76-0.93)$ & $<0.01$ \\
\hline $\mathrm{T} 3$ & $2.66(1.32-5.36)$ & 0.01 & $3.06(1.32-7.08)$ & 0.01 & $3.11(0.77-12.46)$ & 0.11 & $2.75(0.68-11.14)$ & 0.16 & $0.96(0.89-1.03)$ & 0.26 & $0.99(0.91-1.07)$ & 0.81 \\
\hline $\mathrm{T} 4$ & $2.85(1.30-6.26)$ & 0.01 & $2.20(0.74-6.52)$ & 0.16 & $4.39(1.09-17.60)$ & 0.04 & $3.58(0.88-14.52)$ & 0.07 & $1.29(1.16-1.44)$ & $<0.01$ & $1.14(1.02-1.27)$ & 0.02 \\
\hline Unspecific & $3.07(1.45-6.50)$ & $<0.01$ & $4.80(1.56-14.73)$ & 0.01 & $4.45(1.11-17.85)$ & 0.04 & $3.26(0.80-13.25)$ & 0.10 & $1.73(1.57-1.92)$ & $<0.01$ & $1.28(1.15-1.43)$ & $<0.01$ \\
\hline \multicolumn{13}{|l|}{ Nstage } \\
\hline NO & reference & & reference & & reference & & reference & & reference & & reference & \\
\hline N1 & $1.00(0.61-1.64)$ & 0.99 & $1.17(0.62-2.20)$ & 0.62 & $0.95(0.89-1.02)$ & 0.18 & $0.93(0.87-1.01)$ & 0.09 & $1.02(0.96-1.09)$ & 0.43 & $1.13(1.06-1.21)$ & $<0.01$ \\
\hline N2 & $0.46(0.06-3.37)$ & 0.44 & $0.28(0.03-2.75)$ & 0.27 & $1.09(0.89-1.34)$ & 0.39 & $1.03(0.84-1.26)$ & 0.79 & $1.22(1.05-1.42)$ & 0.01 & $1.39(1.19-1.62)$ & $<0.01$ \\
\hline Unspecific & $1.47(0.64-3.37)$ & 0.36 & $0.43(0.12-1.63)$ & 0.22 & $1.53(1.34-1.75)$ & $<0.01$ & $1.31(1.14-1.51)$ & $<0.01$ & $1.99(1.77-2.25)$ & $<0.01$ & $1.42(1.24-1.62)$ & $<0.01$ \\
\hline \multicolumn{13}{|c|}{ Primary site surgery } \\
\hline Yes & $0.33(0.16-0.69)$ & $<0.01$ & $0.06(0.00-0.74)$ & 0.03 & $0.58(0.53-0.63)$ & $<0.01$ & $0.66(0.55-0.80)$ & $<0.01$ & $0.51(0.48-0.54)$ & $<0.01$ & $0.60(0.51-0.69)$ & $<0.01$ \\
\hline No & reference & & reference & & reference & & reference & & reference & & reference & \\
\hline \multicolumn{13}{|c|}{ Lymph node dissection } \\
\hline Yes & $0.36(0.17-0.76)$ & 0.01 & $4.23(0.33-54.23)$ & 0.27 & $0.59(0.53-0.65)$ & $<0.01$ & $0.93(0.76-1.13)$ & 0.45 & $0.53(0.5-0.57)$ & $<0.01$ & $0.90(0.77-1.04)$ & 0.15 \\
\hline No & reference & & reference & & reference & & reference & & reference & & reference & \\
\hline
\end{tabular}

\section{Exploration of the risk factors for overall survival}

Based on the different clinicopathologic features of the different pathological types of tumors and based on the distinct survival rates between metastatic and non-metastatic disease, we explored the risk factors according to the pathological tumor types and metastasis status. We performed univariate and multivariate Cox regression analyses and have presented the results in Tables 1 and 2. Table 1 summarizes the risk factors of non-metastatic disease based on pathologic type, and Table 2 summarizes the metastasis information. For non-metastatic NECs, the risk of death increased with the depth of tumor invasion, while a lower location of the primary site in the esophagus was associated with better survival. Tumor size bigger than 8 centimeters will obviously increase the cancer death risk $(\mathrm{HR}=3.77,95 \% \mathrm{CI}$ : 1.04-13.68, $P=0.04)$. Primary site surgery will bring benefits to this group of patients $(\mathrm{HR}=0.06,95 \% \mathrm{CI}$ : $0.00-0.74, P=0.03)$, while lymphadenectomy at the primary site was not associated with an increased survival benefit. For metastatic disease, liver metastasis was an independent risk factor of NECs (HR=1.68, 95\% CI: 1.05-2.67, $P=0.03$ ). None of the patients with NECs underwent surgery of the 
metastatic sites, so we could not analyze this feature and neither surgery nor lymphadenectomy at primary site was associated with an increased survival benefit. For SCC, in patients older than 66 years, the tumors originated from the lower parts of the esophagus and tumors larger than $7 \mathrm{~cm}$ increased the risk of cancer death. Both metastatic and non-metastatic disease showed benefits from primary tumor surgery $(\mathrm{HR}=0.66,95 \% \mathrm{CI}: 0.51-0.85, P<0.01 ; \mathrm{HR}=0.66,95 \% \mathrm{CI}$ : $0.55-0.80, P<0.01$, respectively), whereas no benefits were associated with radical lymphadenectomy in non-metastatic disease or surgery of the metastatic sites in metastatic disease. In the metastatic disease of SCC, men had a higher risk of death than women, and poor differentiation and the depth of tumor invasion increased the risk of death. With the exception of metastasis to the brain, SCC metastasis to the liver, lung and bone increased the risk of death. For ACs, women had a lower risk of death than men for non-metastatic disease. Moreover, in patients older than 66 years, the tumors originated from the lower parts of the esophagus, the tumors were larger than 2 $\mathrm{cm}$, and the depth of tumor invasion and regional lymph node invasion increased the risk of tumor death. Both metastatic and non-metastatic disease were associated with benefits from primary tumor surgery $(\mathrm{HR}=0.64,95 \% \mathrm{CI}: 0.56-0.73, P<0.01 ; \mathrm{HR}=0.60$, $95 \%$ CI: $0.51-0.69, P<0.01$, respectively). However, no benefits were associated with either radical lymphadenectomy in non-metastatic disease or surgery of the metastatic sites in metastatic disease. $\mathrm{AC}$ metastasis to the liver, lung, bone and brain increased the risk of death.

\section{Exploration of the metastasis pattern}

Different survival rates were associated with the different pathological types, and different metastatic sites contributed varying levels of risk for cancer
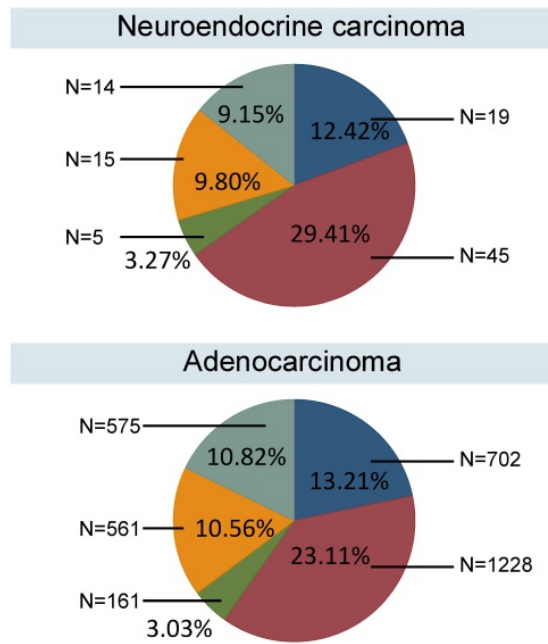

death. Therefore, we have summarized the metastasis patterns of the three pathological types in Fig. 3. The NECs and ACs showed obviously higher rates of metastasis to liver $(29.41 \%$ and $23.11 \%$, respectively), whereas the SCCs typically metastasized to the lung (15.84\%) and distant lymph nodes (15.37\%).

\section{Discussion}

This study is the first to compare esophageal NECs, ACs and SCCs from the same period based on demography. Our study found that the prevalence of ACs surpassed that of SCCs from 2004-2014, which was consistent with recent reports. Melina Arnold et $\mathrm{al}^{16}$ predicted that the future burden of esophageal cancers, especially ACs, would rise dramatically across high-income countries; however, the data describing NECs was sparse. Although esophageal NECs, which reportedly comprise $0.05 \%-7.60 \%$ of esophageal cancers, are rare ${ }^{17}$, NECs have gradually attracted the attention of scientists and clinicians. The prevalence has risen over the past 10 years, and this increase may be attributable to the gradual understanding of NECs by pathologists. As mentioned above, an accurate pathological diagnosis of NECs, although critical for patient management, is challenging due to difficulties in distinguishing NECs from other poorly differentiated esophageal malignant neoplasms. NECs have often been misdiagnosed as SCCs because synchronous SCCs in NECs are also common and because the squamous component often overlies NECs, as first described by McKeon in 1952 and subsequently confirmed by other investigators ${ }^{17-20}$. When intermingled with NECs, an SCC aggregate/pearl is frequently present in the center of the tumor, with undifferentiated neuroendocrine cells at the periphery, thereby showing a dedifferentiation growth pattern ${ }^{17}$.

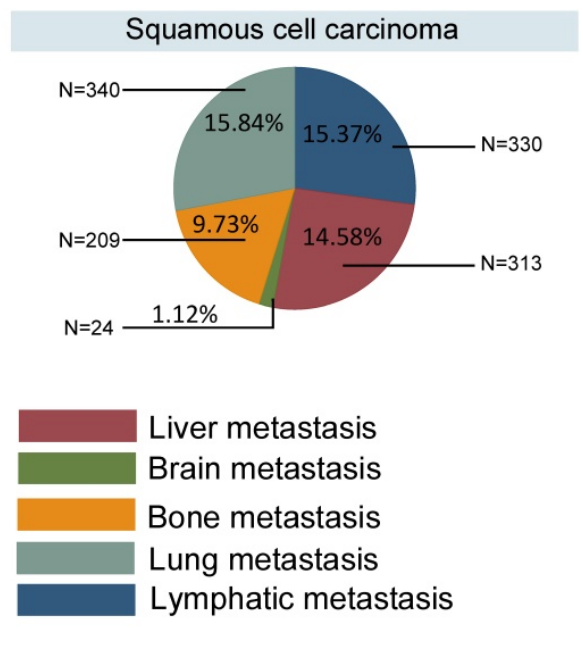

Figure 3. Metastasis patterns in different pathological types. 
Table 2. Risk factors of metastasis disease

\begin{tabular}{|c|c|c|c|c|c|c|c|c|c|c|c|c|}
\hline & \multicolumn{4}{|c|}{ Neuroendocrine carcinoma } & \multicolumn{4}{|c|}{ Squamous cell carcinoma } & \multicolumn{4}{|c|}{ Adenocarcinoma } \\
\hline & \multicolumn{2}{|c|}{ Univariate analyses } & \multicolumn{2}{|c|}{ Multivariate analyses } & \multicolumn{2}{|c|}{ Univariate analyses } & \multicolumn{2}{|c|}{ Multivariate analyses } & \multicolumn{2}{|c|}{ Univariate analyses } & \multicolumn{2}{|c|}{ Multivariate analyses } \\
\hline & $\begin{array}{l}\text { HR } \\
(95 \% \mathrm{CI})\end{array}$ & $\begin{array}{l}\mathrm{P}- \\
\text { value }\end{array}$ & $\begin{array}{l}\text { HR } \\
(95 \% \mathrm{CI})\end{array}$ & $\begin{array}{l}\mathrm{P}- \\
\text { value }\end{array}$ & $\begin{array}{l}\text { HR } \\
(95 \% \mathrm{CI})\end{array}$ & $\begin{array}{l}\mathrm{P}- \\
\text { value }\end{array}$ & $\begin{array}{l}\text { HR } \\
(95 \% \mathrm{CI})\end{array}$ & $\begin{array}{l}P- \\
\text { value }\end{array}$ & $\begin{array}{l}\text { HR } \\
(95 \% \mathrm{CI})\end{array}$ & $\begin{array}{l}\mathrm{P}- \\
\text { value }\end{array}$ & $\begin{array}{l}\text { HR } \\
(95 \% C I)\end{array}$ & $\begin{array}{l}\mathrm{P}- \\
\text { value }\end{array}$ \\
\hline \multicolumn{13}{|l|}{ Gender } \\
\hline Female & Reference & & Reference & & Reference & & Reference & & Reference & & Reference & \\
\hline Male & $0.99(0.66-1.49)$ & 0.96 & $0.92(0.57-1.50)$ & 0.75 & $1.16(1.04-1.28)$ & 0.01 & $1.15(1.03-1.27)$ & 0.01 & $0.93(0.85-1.01)$ & 0.09 & $0.95(0.87-1.03)$ & 0.23 \\
\hline \multicolumn{13}{|l|}{ Age } \\
\hline$<66$ year-old & Reference & & Reference & & Reference & & Reference & & Reference & & Reference & \\
\hline$>=66$ year-old & $1.17(0.83-1.66)$ & 0.37 & $1.42(0.92-2.18)$ & 0.11 & $1.04(0.95-1.14)$ & 0.41 & $1.07(0.97-1.17)$ & 0.17 & $1.21(1.14-1.28)$ & $<0.01$ & $1.19(1.12-1.26)$ & $<0.01$ \\
\hline \multicolumn{13}{|c|}{ Location } \\
\hline $\begin{array}{l}\text { Upper third of } \\
\text { esophagus }\end{array}$ & Reference & & Reference & & Reference & & Reference & & Reference & & Reference & \\
\hline $\begin{array}{l}\text { Middle third } \\
\text { of esophagus }\end{array}$ & $1.33(0.50-3.51)$ & 0.57 & $1.12(0.39-3.20)$ & 0.83 & $0.96(0.83-1.11)$ & 0.57 & $1.01(0.87-1.17)$ & 0.88 & $1.36(0.98-1.90)$ & 0.07 & $1.27(0.91-1.77)$ & 0.16 \\
\hline $\begin{array}{l}\text { Lower third of } \\
\text { esophagus }\end{array}$ & $1.15(0.46-2.84)$ & 0.77 & $1.15(0.40-3.30)$ & 0.79 & $1.02(0.88-1.18)$ & 0.82 & $1.07(0.92-1.24)$ & 0.39 & $1.00(0.73-1.37)$ & 1.00 & $0.98(0.72-1.35)$ & 0.92 \\
\hline Unspecific & $1.27(0.49-3.33)$ & 0.62 & $1.06(0.37-3.06)$ & 0.92 & $1.16(0.98-1.36)$ & 0.08 & $1.13(0.96-1.33)$ & 0.15 & $1.23(0.89-1.70)$ & 0.21 & $1.12(0.81-1.55)$ & 0.49 \\
\hline \multicolumn{13}{|l|}{ Grade } \\
\hline I & Reference & & Reference & & Reference & & Reference & & Reference & & Reference & \\
\hline II & $0.91(0.08-10.14)$ & 0.94 & $0.88(0.08-10.23)$ & 0.92 & $1.21(0.86-1.71)$ & 0.28 & $1.24(0.88-1.76)$ & 0.22 & $1.00(0.83-1.20)$ & 0.99 & $1.02(0.85-1.23)$ & 0.80 \\
\hline III & $0.77(0.11-5.57)$ & 0.79 & $0.52(0.07-3.98)$ & 0.53 & $1.24(0.88-1.75)$ & 0.21 & $1.25(0.89-1.76)$ & 0.20 & $1.26(1.06-1.50)$ & 0.01 & $1.31(1.10-1.57)$ & $<0.01$ \\
\hline IV & $0.59(0.08-4.39)$ & 0.60 & $0.38(0.05-3.02)$ & 0.36 & $2.20(1.18-4.11)$ & 0.01 & $2.21(1.18-4.14)$ & 0.01 & $1.21(0.91-1.62)$ & 0.20 & $1.28(0.96-1.72)$ & 0.09 \\
\hline Unspecific & $1.00(0.14-7.27)$ & 1.00 & $0.67(0.09-5.17)$ & 0.70 & $1.31(0.92-1.86)$ & 0.14 & $1.25(0.88-1.78)$ & 0.22 & $1.12(0.93-1.35)$ & 0.23 & $1.08(0.90-1.30)$ & 0.43 \\
\hline \multicolumn{13}{|l|}{ Tstage } \\
\hline T0 & Reference & & Reference & & Reference & & Reference & & Reference & & Reference & \\
\hline $\mathrm{T} 1$ & NA & & NA & & $0.23(0.06-0.92)$ & 0.04 & $0.27(0.07-1.10)$ & 0.07 & $0.27(0.04-1.94)$ & 0.19 & $0.24(0.03-1.73)$ & 0.16 \\
\hline $\mathrm{T} 2$ & $0.52(0.21-1.25)$ & 0.15 & $0.55(0.20-1.46)$ & 0.23 & $0.19(0.05-0.79)$ & 0.02 & $0.24(0.06-1.00)$ & 0.05 & $0.19(0.03-1.39)$ & 0.10 & $0.19(0.03-1.39)$ & 0.10 \\
\hline T3 & $0.74(0.41-1.34)$ & 0.32 & $0.71(0.36-1.41)$ & 0.33 & $0.17(0.04-0.69)$ & 0.01 & $0.22(0.05-0.88)$ & 0.03 & $0.20(0.03-1.43)$ & 0.11 & $0.20(0.03-1.41)$ & 0.11 \\
\hline $\mathrm{T} 4$ & $0.97(0.59-1.59)$ & 0.91 & $1.04(0.60-1.81)$ & 0.89 & $0.27(0.07-1.09)$ & 0.07 & $0.33(0.08-1.32)$ & 0.12 & $0.28(0.04-2.02)$ & 0.21 & $0.26(0.04-1.84)$ & 0.18 \\
\hline Unspecific & $1.10(0.69-1.76)$ & 0.68 & $0.88(0.49-1.59)$ & 0.68 & $0.24(0.06-0.96)$ & 0.04 & $0.27(0.07-1.08)$ & 0.06 & $0.29(0.04-2.05)$ & 0.21 & $0.24(0.03-1.71)$ & 0.15 \\
\hline \multicolumn{13}{|l|}{ Nstage } \\
\hline No & Reference & & Reference & & Reference & & Reference & & Reference & & Reference & \\
\hline N1 & $0.76(0.50-1.17)$ & 0.22 & $0.71(0.44-1.14)$ & 0.16 & $0.90(0.81-1.01)$ & 0.07 & $0.91(0.81-1.01)$ & 0.09 & $0.95(0.89-1.02)$ & 0.18 & $1.01(0.94-1.08)$ & 0.88 \\
\hline N2 & $0.56(0.17-1.84)$ & 0.34 & $1.21(0.31-4.77)$ & 0.79 & $1.04(0.82-1.32)$ & 0.75 & $0.96(0.75-1.23)$ & 0.76 & $0.92(0.80-1.07)$ & 0.29 & $0.97(0.83-1.13)$ & 0.69 \\
\hline Unspecific & $1.02(0.61-1.70)$ & 0.95 & $1.02(0.57-1.83)$ & 0.95 & $1.08(0.93-1.26)$ & 0.31 & $1.05(0.90-1.23)$ & 0.54 & $1.20(1.10-1.32)$ & $<0.01$ & $1.17(1.06-1.29)$ & $<0.01$ \\
\hline \multicolumn{13}{|c|}{ Distant metastasis sites dissection } \\
\hline Yes & NA & & NA & & $1.00(0.25-3.99)$ & 1.00 & $1.51(0.37-6.08)$ & 0.57 & $0.63(0.32-1.27)$ & 0.20 & $0.98(0.49-1.98)$ & 0.96 \\
\hline No & Reference & & Reference & & Reference & & Reference & Reference & Reference & & Reference & \\
\hline \multicolumn{13}{|c|}{ Primary site surgery } \\
\hline Yes & $0.55(0.20-1.52)$ & 0.25 & $0.82(0.26-2.61)$ & 0.74 & $0.59(0.45-0.75)$ & $<0.01$ & $0.66(0.51-0.85)$ & $<0.01$ & $0.53(0.47-0.60)$ & $<0.01$ & $0.64(0.56-0.73)$ & $<0.01$ \\
\hline No & Reference & & Reference & & Reference & & Reference & & Reference & & Reference & \\
\hline \multicolumn{13}{|c|}{ Liver metasatasis } \\
\hline Yes & $1.77(1.20-2.62)$ & $<0.01$ & $1.68(1.05-2.67)$ & 0.03 & 1.36(1.19-1.56) & $<0.01$ & $1.19(1.03-1.37)$ & 0.02 & $1.30(1.21-1.39)$ & $<0.01$ & $1.15(1.07-1.24)$ & $<0.01$ \\
\hline No & Reference & & Reference & & Reference & & Reference & & Reference & & Reference & \\
\hline Lung metastas & & & & & & & & & & & & \\
\hline Yes & $2.13(1.10-4.15)$ & 0.03 & $1.64(0.66-4.06)$ & 0.29 & $1.34(1.18-1.52)$ & $<0.01$ & $1.20(1.04-1.37)$ & 0.01 & $1.41(1.28-1.55)$ & $<0.01$ & $1.25(1.13-1.39)$ & $<0.01$ \\
\hline No & Reference & & Reference & & Reference & & Reference & & Reference & & Reference & \\
\hline Brain metastas & & & & & & & & & & & & \\
\hline Yes & $1.94(0.71-5.30)$ & 0.20 & $1.10(0.36-3.33)$ & 0.86 & $1.39(0.89-2.16)$ & 0.14 & $1.07(0.68-1.68)$ & 0.77 & $1.41(1.19-1.67)$ & $<0.01$ & $1.26(1.06-1.49)$ & 0.01 \\
\hline No & Reference & & Reference & & Reference & & Reference & & Reference & & Reference & \\
\hline Bone metastas & & & & & & & & & & & & \\
\hline Yes & $1.88(1.06-3.32)$ & 0.03 & $1.40(0.67-2.92)$ & 0.37 & $1.52(1.30-1.78)$ & $<0.01$ & $1.45(1.23-1.70)$ & $<0.01$ & $1.38(1.26-1.52)$ & $<0.01$ & $1.24(1.13-1.37)$ & $<0.01$ \\
\hline No & Reference & & Reference & & Reference & & Reference & & Reference & & Reference & \\
\hline
\end{tabular}

According to the clinical pathological data that we retrieved from SEER, SCCs often originate from the mid-thoracic esophagus, while ACs are often located in the lower thoracic esophagus, which is consistent with previous studies. However, the sites of origin of NECs vary depending on race. Chinese ${ }^{21}$, 22 and Korean ${ }^{23}$ investigators reported previously that NECs were present in the middle of the esophagus, whereas most case reports on Americans have indicated that the majority of NEC tumors are centered in the distal esophagus and are associated with Barrett's esophagus-related diseases ${ }^{24,} 25$. Therefore, we hypothesis that the primary sites differ depending on race. Our data was retrieved from the SEER database, in which white people constituted the majority of patients, so the distal esophagus was the most common site of NECs in our results. Our hypothesis regarding race, however, remains to be tested further in larger studies. For the age of diagnosis, no significant differences were evident among the three groups. In terms of the degree of differentiation, most of the NECs were poorly 
differentiated, and the SCCs and ACs were moderately differentiated. Regarding tumor size, NECs were larger than ACs and SCCs from the same period. The depth of invasion of the NECs was significantly deeper than those of the SCCs or ACs. The proportion of NEC patients with metastases was significantly higher than the proportions of SCC and AC patients. The obviously worse condition at the time of diagnosis for the NEC group might be heavily related to a lack of understanding by clinicians and the limited methods for the early diagnosis of NECs.

Based on the clinical pathological characteristics, one can easily imagine that the prognosis of NECs would be worse than the prognosis of SCCs or ACs, and this notion is also demonstrated in our study. The median overall survival and five-year survival rates of NECs are significantly worse than those of contemporaneous ACs, but no statistical significance is evident for SCCs. Systemic metastasis is the leading cause of patient death, and therefore, our study divides the patients into metastasis and non-metastasis groups for further analysis and to identify risk factors of cancer death. Meanwhile, the metastasis spectra of the three histological types of esophageal cancer are summarized for clinicians and scientific researchers, with the hope that these data will provide additional ideas for studies of the distinct biological behaviors of these tumors. However, more accurate data based on larger sample sizes are required for further study. In the non-metastatic group, the depth of tumor invasion, level of differentiation and primary tumor sites were correlated with the prognosis of NECs; age, the primary tumor site, and tumor size were correlated with the prognosis of SCCs; and age, the primary tumor site, tumor size, the depth of invasion, and in situ lymph node infiltration were all correlated with the prognosis of ACs. Notably, for non-metastatic ACs, males had a better prognosis than females. In the metastatic group, we have described the metastatic sites in detail. We found that for NECs, hepatic metastasis was an independent prognostic risk factor. For SCCs, men had a poorer survival rate than women, and liver, lung, and bone metastases were predictive factors of poor prognosis. For ACs, an age above 66-years increased the risk of cancer death, and all metastasis types were independent prognostic factors.

According to the NCCN guidelines ${ }^{26}$, radical operation is recommended for locational esophageal cancer, and systematic therapy is advocated for metastatic disease. In this study, we further analyzed the value of radical surgery, lymph node dissection and metastatic site resection for the different pathological types. The results indicated that surgery of the primary site and lymph node dissection could not produce survival benefits for NECs when developed the metastatic disease. However, primary site surgery produced survival benefits for SCCs and ACs. However, benefits from lymph node dissection and distant metastatic site resection were not observed for SCCs and ACs in our study.

Due to the low incidence of esophageal NECs and the lack of related clinical studies, there are no unique staging and grading systems and guidelines for clinicians to select proper strategies for patients. The treatment of patients is mostly performed according to the personal experience of the clinician. Therefore, the value of our study is apparent. We have concentrated the available clinical data, which will lay the foundation for clinical guidelines. We have observed that patients with NECs don't benefit from surgery and that these patients show a high incidence of metastatic disease. Thus, systematic therapy might be the first-line therapy for patients with NECs. For patients with ACs and SCCs, surgical therapy is still the best choice.

Our study has several limitations. First, due to the absence of information regarding chemotherapy and radiotherapy in the SEER database, the effects of chemotherapy on survival could not be evaluated. Second, in the SEER database, only metastases to the following sites were included: bone, lung, liver, brain and distant lymph node. Third, the SEER database does not contain pathological data, such as Ki-67 scores, which are recommended for the new staging and grading system of neuroendocrine carcinomas. Thus, the limitations noted above impose the restrictions on further studies.

\section{Conclusion}

NEC, SCC and AC are the three main pathological types of esophageal carcinoma, and few studies have focused on the differences between them. The prevalence of ACs was higher than that of SCC from 2004-2014, and the prevalence of NECs is increasing. The clinicopathological features and the factors affecting patient survival vary between the pathological types. NECs are associated with an apparently worse survival rate than SCCs and ACs, while ACs have the best prognosis. The metastasis patterns are different between NECs, SCCs and ACs, which suggests distinct biological behaviors. Patients with SCCs and ACs can obviously obtain benefits from primary site surgery regardless of the metastasis status, but the benefits are not significant for patients with NECs. The benefits of lymph node dissection and metastasis dissection for patients with NECs, ACs and SCCs need further exploration. The apparent distinctions above are important for tumor staging 
and treatment selection. Therefore, we advocate a unique staging and grading system for esophageal NECs to guiding clinicians toward making better choices for patients.

\section{Supplementary Material}

Supplementary figures and tables. http:/ / www.jcancer.org/v10p1520s1.pdf

\section{Acknowledgments}

Key Projects in the National Science \& Technology Pillar Program during the Twelfth Five-year Plan Period (No. 2014BAI09B07), National Human Genetic Resources Sharing Service Platform (2005DKA21300), the National Key R\&D Program of China (2017YFC0 908200) and National Natural Science Foundation of China (81472664 \& 81772545).

\section{Competing Interests}

The authors have declared that no competing interest exists.

\section{References}

1. Siegel RL, Miller KD, Jemal A. Cancer Statistics, 2017. CA Cancer J Clin 2017;67:7-30.

2. Chen $W$, Zheng R, Baade PD, Zhang S, Zeng H, Bray F, Jemal A, Yu XQ, He J. Cancer statistics in China, 2015. CA Cancer J Clin 2016;66:115-32.

3. Siegel R, DeSantis C, Virgo K, Stein K, Mariotto A, Smith T, Cooper D, Gansler $\mathrm{T}$, Lerro C, Fedewa S, Lin C, Leach C, et al. Cancer treatment and survivorship statistics, 2012. CA Cancer J Clin 2012;62:220-41.

4. Torre LA, Bray F, Siegel RL, Ferlay J, Lortet-Tieulent J, Jemal A. Global cancer statistics, 2012. CA Cancer J Clin 2015;65:87-108.

5. Siegel R, Naishadham D, Jemal A. Cancer statistics, 2012. CA Cancer J Clin 2012;62:10-29.

6. Arnold M, Soerjomataram I, Ferlay J, Forman D. Global incidence of oesophageal cancer by histological subtype in 2012. GUT 2015;64:381-7.

7. McKeown F. Oat-cell carcinoma of the oesophagus. J Pathol Bacteriol 1952;64:889-91.

8. Chen SB, Yang JS, Yang WP, Weng HR, Li H, Liu DT, Chen YP. Treatment and prognosis of limited disease primary small cell carcinoma of esophagus. DIS ESOPHAGUS 2011;24:114-9.

9. Law SY, Fok M, Lam KY, Loke SL, Ma LT, Wong J. Small cell carcinoma of the esophagus. CANCER-AM CANCER SOC 1994;73:2894-9.

10. Li AF, Hsu HS, Hsu CY, Li AC, Li WY, Liang WY, Chen JY. A 20-year retrospective study of small-cell carcinomas in Taiwan. J SURG ONCOL 2010;102:497-502.

11. Li AF, Li AC, Hsu CY, Li WY, Hsu HS, Chen JY. Small cell carcinomas in gastrointestinal tract: immunohistochemical and clinicopathological features. J CLIN PATHOL 2010;63:620-5.

12. Hosokawa A, Shimada Y, Matsumura Y, Yamada Y, Muro K, Hamaguchi T, Igaki H, Tachimori Y, Kato H, Shirao K. Small cell carcinoma of the esophagus. Analysis of 14 cases and literature review. Hepatogastroenterology 2005;52:1738-41.

13. Mitani M, Kuwabara Y, Shinoda N, Sato A, Fujii Y. Long-term survivors after the resection of limited esophageal small cell carcinoma. DIS ESOPHAGUS 2000;13:259-61.

14. Bennouna J, Bardet E, Deguiral P, Douillard JY. Small cell carcinoma of the esophagus: analysis of 10 cases and review of the published data. Am J Clin Oncol 2000;23:455-9.

15. Ku GY, Minsky BD, Rusch VW, Bains M, Kelsen DP, Ilson DH. Small-cell carcinoma of the esophagus and gastroesophageal junction: review of the Memorial Sloan-Kettering experience. ANN ONCOL 2008;19:533-7.

16. Arnold M, Laversanne M, Brown LM, Devesa SS, Bray F. Predicting the Future Burden of Esophageal Cancer by Histological Subtype: International Trends in Incidence up to 2030. AM J GASTROENTEROL 2017;112:1247-55.

17. Huang Q, Wu H, Nie L, Shi J, Lebenthal A, Chen J, Sun Q, Yang J, Huang L, Ye Q. Primary high-grade neuroendocrine carcinoma of the esophagus: a clinicopathologic and immunohistochemical study of 42 resection cases. AM J SURG PATHOL 2013;37:467-83.

18. McFadden DW, Rudnicki M, Talamini MA. Primary small cell carcinoma of the esophagus. ANN THORAC SURG 1989;47:477-80.
19. Briggs JC, Ibrahim NB. Oat cell carcinomas of the oesophagus: a clinico-pathological study of 23 cases. HISTOPATHOLOGY 1983;7:261-77.

20. Huang Q, Fang DC, Yu CG, Zhang J, Chen MH. Barrett's esophagus-related diseases remain uncommon in China. J Dig Dis 2011;12:420-7.

21. Zhao E, Ling T, Xu J, Zhao G, Cao H, Giacopuzzi S, Bencivenga M, de Manzoni G. Turning left or right? A comparative analysis in adenocarcinomas of the esophagogastric junction according to the seventh AJCC TNM classification for cancers of the esophagus and stomach: experience in a Chinese single institution. INT J CLIN EXP MED 2015;8:10668-77.

22. Wu Z, Ma JY, Yang JJ, Zhao YF, Zhang SF. Primary small cell carcinoma of esophagus: report of 9 cases and review of literature. World J Gastroenterol 2004;10:3680-2.

23. Suh YS, Han DS, Kong SH, Lee HJ, Kim YT, Kim WH, Lee KU, Yang HK. Should adenocarcinoma of the esophagogastric junction be classified as esophageal cancer? A comparative analysis according to the seventh AJCC TNM classification. ANN SURG 2012;255:908-15.

24. Huang $\mathrm{Q}$, Shi J, Sun $\mathrm{Q}$, Fan X, Feng A, Wu H, Zhou Q, Yu C, Mashimo H, Lauwers GY. Distal esophageal carcinomas in Chinese patients vary widely in histopathology, but adenocarcinomas remain rare. HUM PATHOL 2012;43:2138-48.

25. Huang Q, Fang DC, Yu CG, Zhang J, Chen MH. Barrett's esophagus-related diseases remain uncommon in China. J Dig Dis 2011;12:420-7.

26. Ajani JA, D'Amico TA, Almhanna K, Bentrem DJ, Besh S, Chao J, Das P, Denlinger C, Fanta P, Fuchs CS, Gerdes H, Glasgow RE, et al. Esophageal and esophagogastric junction cancers, version 1.2015. J Natl Compr Canc Netw 2015;13:194-227. 